\title{
The Application Status and Development Trend of Digital Art Sensory Interaction
}

\author{
Hongyan Ren* \\ Chengdu Art Vocational University, Chengdu, Sichuan 610000, China.
}

\begin{abstract}
: with the continuous development of digital media, the high-frequency interaction between people and personal digital devices continues to promote the development of digital entertainment products. At present, digital art has its own development in the interaction of vision, hearing, touch, taste and smell. This article summarizes the application status of sensory interaction, analyzes the future development trend of digital art, and finds out that digital art will develop in the fields of artificial intelligence, sensory equipment and sensor technology iteration in the future through data analysis, literature study and case analysis In this paper, the author come up with some feasible reference for practitioners and researchers in related fields.
\end{abstract}

Keywords: Digital Art; Digital Entertainment; Sensory Interaction

Since the popularity of personal digital devices represented by computers and smart phones, the vigorous development of digital media has made various relatively independent media systems of human civilization entering the stage of integrated communication. The organic combination of words, images and sounds has greatly improved the efficiency of information dissemination. Human beings can interact with these information elements through different behaviors, even if they are not In different physical spaces, similar face-to-face negotiation activities can still be carried out. The birth and popularization of digital media is no longer a single technological breakthrough. Just as Negator said, human society has quietly carried out a profound social change and stepped into the mode of "digital survival".

Digital media plays a very important role in modern people's life. The data released by China Internet Network Information Center (CNNIC) shows that the average weekly online time of Chinese Internet users has been increasing in the past six years. As of March 2020, the average weekly online time is 30.8 hours, an increase of 3.2 hours compared with the end of 2018 . If you add in the length of the computer, the number will be doubled. The high frequency and long-term interaction between people and digital media has promoted the rapid development of digital entertainment products, and many new forms of artistic creation have been born in the context of digital media. This paper will study and discuss the current situation and development trend of sensory interaction application in digital art creation.

\section{Review of media development}

After hundreds of thousands of years of evolution, ancient Homo sapience has evolved a complete set of human internal information system. The eyes, ears, mouth, nose and skin collect information, the nervous system transmits information, and the brain judges and processes information and issues feedback instructions. The cooperative labor of human beings to create civilization needs frequent interpersonal communication. As the first set of systematic externalized symbol information transmission system, language emerges as the times are required. Although the emergence of language has greatly promoted the development of human society, sound information has timeliness and transience, which cannot be effectively recorded under the material conditions and technological level at that time. The invention of characters made up for the defects of language symbols. The inheritance of civilization no longer depended on the word of mouth and super memory of historians. However, before the advent of printing, characters were monopolized by a few upper classes, and the literacy rate of ordinary people was still very low. It was not until Gutenberg made a modern mechanical printer according to Chinese movable type printing that the mass reproduction of characters was realized, and the character symbols were really popularized to all social classes. However, due to the fact that the propagation of atoms depended on material carriers, the speed of information transmission at that time was still very slow, which is almost equal to the speed of vehicles, horses, cargo ships and other means of transportation.

With the development of modern science and technology, the birth of radio and cable technology marks the arrival of the era of mass communication. With the advent of wireless broadcasting and cable TV, the speed of information transmission is close to the speed of sound and light. As the first equipment to combine audio and visual functions, TV has rapidly become the backbone of mass communication. Audio visual art has become the youngest and most influential art form ${ }^{[1]}$.

In the 1990s, the price of electronic computers gradually declined, and ordinary people also had the opportunity to have close

Copyright (C) 2020 Hongyan Ren

doi: $10.18282 / 1-e . v 9 i 4.1708$

This is an open-access article distributed under the terms of the Creative Commons Attribution Non-Commercial License (http://creativecommons.org/licenses/by-nc/4.0/), which permits unrestricted non-commercial use, distribution, and reproduction in any medium, provided the original work is properly cited. 
contact with bit. The popularity of digital TV, tablet computers and smart phones makes audio-visual entertainment products enter the home interactive mode and greatly promotes the development process of digital life. On the terminal of personal digital devices, words, sounds and images are no longer limited to a single transmission channel. Symbol elements acting on a variety of senses are transmitted interactively in cyberspace. The participants of communication are expanded, the forms of communication are integrated, and the representations of communication are diversified. Today, digital products seem to have become a field of entertainment for both refined and popular tastes, suitable for young and old people, and digital art works with different forms are emerging.

\section{Application status of sensory interaction in digital art creation}

\subsection{Audio visual sensory interaction}

Ninety percent of the information received by the human body comes from vision and hearing. Art creators throughout the ages have been committed to exploring art forms that combine audio-visual functions. From shadow play, puppet show, stage play to television and film, image and sound combine in different ways to create diversified forms of artistic expression. After entering the 20th century, the breakthrough of electronic information technology has made the computer realize the organic integration of audio-visual senses and human-computer interaction, and electronic games have become brilliant in the field of digital entertainment, with the number of players increasing year by year.

Video games, represented by virtual reality games, have been successful in the application of audio-visual sensory interaction. On April 24, 2020 Beijing time, Travis Scott, a famous American rapper, presented a large-scale immersive concert called "astronomic" to the global game players in the virtual reality game "Fortress night". At the beginning of the concert, the virtual simulation image of the singer, like an ancient giant, came down from the sky with the meteorite, bringing an audio-visual feast across the space, land and sea for the game players. So far, more than 27.7 million players have watched it. The combination of immersive audio-visual interaction through virtual reality technology is not only a simple superposition of the two, but also a systematic, related and multilevel stimulation of the two human functions from the information receiving channel system, which can mobilize the overall perception mechanism of audio-visual function system to a large extent.

\subsection{Other sensory interactions}

Audio visual information is transmitted with sound and light as the material carrier. The receiver can complete the communication activities as long as he has sound vision and hearing. Therefore, it is the most convenient and fastest in the developing field of digital art creation. Due to the technical bottleneck, it is difficult to solve, there are relatively few digital art works around skin function, but creators and scientific research staff have been trying to explore these areas.

Skin sensation is tactile. Human nerve cells are scattered on the surface of the skin to sense the temperature, humidity, vibration, pressure, material and other information of the contact, and form a self-protective mechanism by producing pain. Touch is an important perception channel for human beings to recognize foreign objects, especially in the situation where audio-visual senses cannot give full play to their effectiveness, touch will affect the human body's judgment of the environment to a certain extent.

For example, when the movie are played,all kinds of devices on the seats of 4D cinema will simulate the movement change, visual angle change, weather change, environmental change and other information elements with the change of image content and plot, and create Somali-sensory interaction such as shock, water mist, bubble, noise and wind. The superposition of multiple sensory interaction brings a novel viewing experience for the viewer. However, due to the high cost of equipment purchase in the early stage, the ticket price of 4D movie is almost twice as high as that of 3D movie. 4D movies not only require good audio-visual experience, but also must consider the combination of movie plot and somatosensory device node in the production process, so usually this kind of movies play for a short time. At the same time, simple water spraying, shaking and wind making may bring a more novel impression in the first experience, but it is difficult to attract viewers to pay multiple times for the current slightly thin presentation. Due to the above reasons, 4D film has not yet been born. It is still in the stage of exploration and experiment, and the rapid development of its scale and industrialization needs more research by researchers.

Whether in the real world or in the virtual world, olfaction belongs to interval perception, which cannot last for a long time and can interact without touching the object. The development of taste interaction is slower than that of other functions. The main reason is that the premise of taste generation is that the mouth contains actions. Generally, the taste buds can be perceived by the human nervous system only when the physical entities enter the mouth. This characteristic also determines that its safety must be taken as the research object. It is difficult to imagine that an unknown substance enters the oral cavity through a certain device, and the owner will not feel confused and uneasy. Therefore, at present, the research of the two basically exists in the laboratory or experimental works, and there is no representative work in the commercial field. It should be noted that the language way of smell and taste can bring strong sensory stimulation and emotional association, and its related research is likely to be the only way to expand the expression form of digital art in the future.

\section{The future development trend of Digital Art}

Artificial intelligence is human's unique consciousness and behavior to engage in art creation subjectively and actively. Science and technology is an important driving force to promote the development of human society. In the 21st century, human civilization has entered the middle stage of digital development. Art and science and technology are perfectly combined in the digital media platform, forming a comprehensive art form of interactivity and interdisciplinary integration. The information dissemination pattern of digital art is highly consistent with the characteristics of cross-border integration, human-computer interaction and cross 
media collaboration presented by artificial intelligence. The organic combination of technology and artificial intelligence is not only the advantages of both, but also the necessity of development.

Ha mad Amalgam, director of art and artificial intelligence laboratory and professor of computer science at Rutgers University, believes that artificial intelligence will become a tool for artists to create, but artificial intelligence itself is creative as a tool, and artists can get inspiration and expand their imagination from tools. Professor Amalgam's view has certain reference significance for us to grasp the future development trend of digital art. Artificial intelligence has a strong ability of deep learning and integration and imitation, which can save the time spent by artists in creative inspiration and open up creative forms and fields.

Audio visual equipping.

At present, the frontier exploration of audio-visual communication mainly focuses on the production, dissemination and reception of immersive information. The current virtual reality technology usually requires the owner to wear heavy VR glasses, and the body is bound with data collection devices, so the human body is limited in a very limited space. However, with the development of holography and naked eye 3D technology, the sense of visual boundary will be effectively eliminated, and in ear or bone conduction headphones will also solve the problem of sound tracking to a large extent.

Iterative upgrading of sensing technology.

At present, intelligent polymer materials and optoelectronic materials are mainly used in the field of fashion design and production $^{[2]}$. Once their technical barriers are broken, the magic girl in the science fiction film "X-Men" will become a reality. The real-time capture and data exchange of human motion and vibration information by intelligent materials can realize real-time force feedback. Since 2019, there has been a major breakthrough in quantum entanglement technology at home and abroad, and the sensing technology has gradually entered the era of physical limitations in space, and somatosensory interaction will become history.

Here, we might as well imagine the future of our daily life after the maturity and popularization of quantum polymer sensing technology: a beautiful lady gets up, washes and calls the intelligent housekeeper, who chooses the appropriate makeup and clothing from the personal image customization platform according to the schedule of the day, and the clothing materials composed of intelligent materials immediately appear. The combination of intelligent materials and quantum technology makes her step into the public space with perfect image every minute.

To sum up, the creation of digital art has a certain development in vision, hearing, touch, taste and body sense. The digital art in the future will become the daily art. To sum up, the creation of digital art has a certain development in vision, hearing and touch, and the commercialization of the interaction between smell and taste needs further exploration. Under the background of the continuous development of artificial intelligence, the field of digital art will realize the expansion of diversified art expression forms and means. At the same time, the sensor technology of multi-sensory interaction and iterative upgrading after equipping will continue to affect the audience's information receiving language environment. With the development of science and technology, the digital art in the future will become the daily art .

\section{Introduction to the author:}

Hongyan Ren, September 11, 1989, Gender: Female, Nationality: Han, Native place: Chengdu, Sichuan, Education: Master, Title: Lecturer, Research direction: Digital Media Art.

\section{References}

[1]McLuhan. Understanding Media: The Extensions of Man[M].Jiangsu Province: Yilin Translation Publishing House,2011.

[2]Liu Na. The application of smart materials in clothing [J] Shanghai Textile Science \& Technology, 2011, 39(07). 